\title{
The clinical features and prognosis of infective endocarditis in the elderly from 2007 to 2016 in a tertiary hospital in China
}

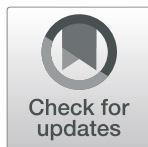

\author{
Zhenzhu Wu ${ }^{\dagger}$, Yi Chen $^{\dagger}$, Tingting Xiao, Tianshui Niu, Qingyi Shi and Yonghong Xiao*
}

\begin{abstract}
Background: Infective endocarditis (IE) especially in the elderly is a serious disease, with a worse prognosis.

Methods: A retrospective cohort study was conducted. A total of 405 patients with definite IE were divided into three groups: 205 patients under 50 years old, 141 patients between 50 and 64 years old and 59 patients over 65 years old.

Results: For older patients, clinical symptoms such as fever, anemia, and heart murmur were as common as the younger patients. IE in old patients had more frequent nosocomial origin $(P=0.007)$ and tended to be more frequent with bad oral hygiene $(p=0.008)$. The most frequent isolated pathogens in the old groups was streptococci and coagulase-negative staphylococci. The old patients had a lower operation rate $(40.7 \%$ vs $58.9 \%$ vs $62.4 \%$, $P=0.012)$ and higher in-hospital mortality ( $20.3 \%$ vs $10.6 \%$ vs $8.8 \%, P=0.044)$ compared with the younger patients. Surgical treatment was a significant predictor of one-year mortality even after adjusting for the confounders $(H R=2.45$, $95 \% \mathrm{Cl} 1.027-10.598, P=0.009)$. The one-year survival rate was higher for older patients with surgical intervention than those without ( $95.8 \%$ vs $68.6 \%, P=0.007)$.

Conclusions: Older patients with IE presented with more comorbidities, bad oral hygiene, more nosocomial origin and a more severe prognosis than younger patients. Streptococci was the most frequent micro-organisms in this group. Surgery were underused in old patients and those with surgical treatment had better prognosis.
\end{abstract}

Keywords: Infective endocarditis, Older patients, Risk factors, Surgical treatment

\section{Background}

Infective endocarditis (IE) is a severe disease with a high burden of mortality and morbidity [1]. Over the past few decades, with the increase in life span and invasive procedures, IE has become more and more frequent in the elderly $[2,3]$. The increasing age of patients with IE will become the major determinant of disease characteristics in the future. Therefore, it is significantly important to explore the clinical features of old patients with IE at present.

\footnotetext{
* Correspondence: xiaoyonghong@zju.edu.cn

${ }^{\dagger}$ Zhenzhu Wu and Yi Chen contributed equally to this work.

State Key Laboratory for Diagnosis and Treatment of Infectious Diseases, National Clinical Research Center for Infectious Diseases, Collaborative Innovation Center for Diagnosis and Treatment of Infectious Diseases, The First Affiliated Hospital, College of Medicine, Zhejiang University, Hangzhou, China
}

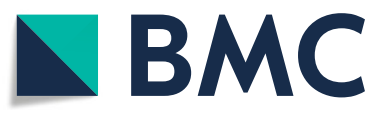

(c) The Author(s). 2019 Open Access This article is distributed under the terms of the Creative Commons Attribution 4.0 International License (http://creativecommons.org/licenses/by/4.0/), which permits unrestricted use, distribution, and reproduction in any medium, provided you give appropriate credit to the original author(s) and the source, provide a link to the Creative Commons license, and indicate if changes were made. The Creative Commons Public Domain Dedication waiver (http://creativecommons.org/publicdomain/zero/1.0/) applies to the data made available in this article, unless otherwise stated. erly is a different disease, with a higher mortality $[2,3]$. The most frequent causative organism as reported previously is Staphylococcus aureus and methicillin-resistant Staphylococcus aureus has a high infection rate in older patients [2, 3]. Previous reports show that clinical presentations of IE in the elderly are often nonspecific and atypical, which often lead to the delay in diagnosis and treatment for this unique population [4-6]. And the proportion of patients undergoing surgical treatment is lower in older patients compared with the younger because of the increased risk caused by aging [7].

However, most of the reports derive from developed countries, and studies exploring the clinical features of older patients with IE in developing countries are scarce. The aim of this article is to investigate the clinical 
features and prognosis of IE in the elderly from a tertiary teaching hospital in east China.

\section{Methods}

\section{Patient selection and study design}

The study was conducted in the First Affiliated Hospital of Zhejiang University, Hangzhou, China. Patients with definite IE from January 1, 2007 to December 31, 2016 were reviewed. All patients were identified according to the modified Duke criteria [8] and patients under 16 years old were excluded. Patients with IE who were admitted to the hospital more than once during the study period were considered as one case. All the patients included were separated into three groups according to their age: 205 patients under 50 years old, 141 patients between 50 and 64 years old and 59 patients over 65 years old. Then, patients over 65 years old were divided into the survival group and the mortality group according to their one-year outcome.

The analysis strategies were as the follows: (1) To analyze the clinical features of IE in the elderly, a comparison between the three groups was conducted; (2) to explore the prognostic risk factors of one-year mortality for older patients and a comparison between the survival group and mortality group was conducted; (3) to evaluate the effect of surgical treatment on older patients, and analysis of the one-year survival rate between patients with antibiotic therapy combined with surgical intervention and patients with antibiotic treatment alone.

\section{Clinical parameters and definition}

The data was obtained from the Electronic Medical Record. The following data were recorded: age, gender, underlying diseases general, IV drug addiction, dental condition and interventions, length of hospitalization, clinical, laboratory and microbiological data, vegetation location, complications of IE, treatments (included antibiotics and surgical intervention), and outcomes.

The modified Duke criteria, which were used to evaluate our patients, involve two major criteria: (1) the presence of at least two positive blood cultures with typical organisms consistent with IE, and (2) evidence of endocardial involvement, primarily diagnosed using echocardiography; and several minor criteria such as predisposing cardiac condition or injection drug abuse, fever $>38^{\circ} \mathrm{C}$, vascular phenomena (arterial embolism, septic pulmonary infarction, intracranial hemorrhage, Janeway lesions) or immunologic phenomena (Osler nodes, Roth spots, glomerulonephritis), and serological evidence of organisms consistent with IE. To be enrolled in this study as definite IE, patients had to meet one of the following criteria: (1) the major two criteria; (2) one major and three minor criteria; (3) five minor criteria; or (4) pathological criteria: microorganisms demonstrated by culture or on histological examination of a vegetation, a vegetation that has embolized, or an intracardiac abscess specimen; or pathological lesions; vegetation or intracardiac abscess by histological examination showing active endocarditishis.

Surgical indications were based on the European Society of Cardiology (ECS) guidelines: heart failure, uncontrolled infection and prevention of embolism were the main indications of surgery $[8,9]$.

The main outcome was one-year all-cause mortality. The one-year follow-up data were collected from the patients' latest visits to our hospital. Transthoracic echocardiogram was performed routinely in all patients. Transesophageal echocardiogram (TEE) was used to detect cases with negative transthoracic echocardiography (TTE) results. Blood culture was performed in all the patients with aerobic, anaerobic and fungal blood cultures, but blood cultures for the HACEK group (Haemophilus spp, Aggregatibacter spp, Cardiobaterium hominis, Eikenella corrodens, and Kingella kingae), and anti-legionella, mycoplasma and bartonella anti-body tests as well as PCR test were not performed when patients had negative blood culture results.

\section{Statistical analysis}

The clinical features analysis was performed using Pearson' $\mathrm{s}$ X2 test or Fisher' $\mathrm{s}$ exact test as appropriate for categorical variables and independent Student's t-test or Rank sum test was used as appropriate for continuous variables. Cox univariate and multivariate survival analysis was performed to discover the predictors of one-year all-cause mortality. A Kaplan-Meier analysis was used to determine the one-year survival. All tests were 2-tailed, and $P<0.05$ was considered statistically significant. All analyses were performed using the SPSS version 23 statistical software.

\section{Results}

\section{Patient enrollment}

Between January 2007 and December 2016, there were 405 patients (male: 64.4\%, age: 16-86) included in our study. Of these patients, 205 patients were in the $<50$ years group, 141 patients in the 50-64 years group and 59 patients in the $\geq 65$ years group. During the follow-up, 92.8\% (378 of 407) of patients taking part in the study completed a median follow-up of one year after infective endocarditis diagnosis.

\section{The clinical characteristics of IE in the three groups}

The clinical characteristics of IE in the three groups are summarized in Table 1.

There was no statistically significant difference between the age groups in terms of the length of hospital stay, duration of symptoms before echocardiography, duration of 
Table 1 Clinical characteristics of patients with infective endocarditis

\begin{tabular}{|c|c|c|c|c|}
\hline Variables $\%$ or Mean \pm SD & $\begin{array}{l}<50 \text { years old } \\
n=205 \\
(50.6 \%)\end{array}$ & $\begin{array}{l}50-64 \text { years old } \\
n=141 \\
(34.8 \%)\end{array}$ & $\begin{array}{l}\geq 65 \text { years old } \\
n=59 \\
(14.6 \%)\end{array}$ & $P$ value \\
\hline \multicolumn{5}{|l|}{ Clinical data } \\
\hline Male & $127(62.0)$ & $93(66.0)$ & $41(69.5)$ & .508 \\
\hline Age & $35 \pm 9$ & $57 \pm 4$ & $72 \pm 5$ & $P<0.001$ \\
\hline Hospital stay & $24 \pm 19$ & $28 \pm 27$ & $23 \pm 19$ & .295 \\
\hline Duration of symptoms before echocardiography & $30 \pm 37$ & $24 \pm 27$ & $24 \pm 34$ & .174 \\
\hline Duration of symptoms before diagnosis & $42 \pm 39$ & $42 \pm 44$ & $42 \pm 58$ & .991 \\
\hline Time interval from diagnosis to surgery & $23.5(8-55.5)$ & $16(6.5-54)$ & $30(11.5-64)$ & .322 \\
\hline Time interval from embolism to diagnosis & $4 \pm 3$ & $13 \pm 17$ & $11 \pm 10$ & .001 \\
\hline Time interval from cerebral embolism to diagnosis & $4 \pm 2$ & $16 \pm 21$ & $13 \pm 12$ & $.006^{*}$ \\
\hline \multicolumn{5}{|l|}{ IE localization } \\
\hline Native valve & $191(93.2)$ & $128(90.8)$ & $49(83.1)$ & .059 \\
\hline Mitral & $61(30.0)$ & $44(31.2)$ & $15(25.4)$ & \\
\hline Aortic & $54(26.3)$ & $56(40.0)$ & $18(30.5)$ & \\
\hline Mitro-aortic & $31(15.1)$ & $17(12.1)$ & $8(13.5)$ & \\
\hline Right valves & $23(11.2)$ & $2(1.4)$ & $4(6.8)$ & \\
\hline Prosthetic valve & $13(6.3)$ & $10(7.1)$ & $8(13.6)$ & .176 \\
\hline Mitral & $4(2.0)$ & $4(2.8)$ & $3(5.1)$ & \\
\hline Aortic & $5(2.4)$ & $4(2.8)$ & $3(5.1)$ & \\
\hline Mitro-aortic & $1(0.5)$ & 0 & $1(1.7)$ & \\
\hline Pacemaker & $1(0.5)$ & $3(2.1)$ & $2(3.4)$ & .191 \\
\hline Community origin & $187(91.2)$ & $124(87.9)$ & $47(79.7)$ & .050 \\
\hline Nosocomial origin & $3(1.5)$ & $6(4.3)$ & $6(10.2)$ & .007 \\
\hline \multicolumn{5}{|l|}{ Comorbidities } \\
\hline \multicolumn{5}{|l|}{ Predisposing cardiac conditions } \\
\hline Rheumatic heart disease & $28(13.7)$ & $30(21.3)$ & $11(18.6)$ & .169 \\
\hline Congenital heart disease & $71(34.6)$ & $23(16.3)$ & $10(16.9)$ & $P<0.001$ \\
\hline Previous cardiac surgery & $16(7.8)$ & $14(9.9)$ & $14(23.7)$ & .002 \\
\hline Degenerative heart disease & $1(0.5)$ & $6(4.3)$ & $6(10.2)$ & .001 \\
\hline Chronic pulmonary disease & $1(0.5)$ & $3(2.1)$ & $2(3.4)$ & .191 \\
\hline History of cancer & $3(1.5)$ & $6(4.3)$ & $2(3.4)$ & .275 \\
\hline Hemodialysis & $3(1.5)$ & $7(5.0)$ & $4(6.8)$ & .069 \\
\hline Liver cirrhosis & $2(1.0)$ & $1(0.7)$ & $1(1.7)$ & .813 \\
\hline Hypertension & $14(6.8)$ & $43(30.5)$ & $25(42.4)$ & $P<0.001$ \\
\hline Diabetes & $2(1.0)$ & $25(17.7)$ & $10(16.9)$ & $P<0.001$ \\
\hline Intravenous drug abuse & $2(1.0)$ & 0 & $1(1.7)$ & .250 \\
\hline Immunodepression & $17(8.3)$ & $12(8.5)$ & $4(6.8)$ & .915 \\
\hline Bad oral hygiene & $57(27.8)$ & $45(31.9)$ & $29(49.2)$ & .008 \\
\hline \multicolumn{5}{|l|}{ Symptoms and signs } \\
\hline Fever & $186(90.7)$ & $125(88.7)$ & $53(89.8)$ & .820 \\
\hline Anemia & $116(56.6)$ & $68(48.2)$ & $38(64.4)$ & .085 \\
\hline Osler nodule & $5(2.4)$ & $1(0.7)$ & $1(1.7)$ & .439 \\
\hline Janeway lesions or nailbed bleeding & $3(1.5)$ & $1(0.7)$ & 0 & .601 \\
\hline New or changing heart murmur & $172(83.9)$ & $110(78.0)$ & $45(76.3)$ & .253 \\
\hline
\end{tabular}


Table 1 Clinical characteristics of patients with infective endocarditis (Continued)

\begin{tabular}{|c|c|c|c|c|}
\hline Variables $\%$ or Mean \pm SD & $\begin{array}{l}<50 \text { years old } \\
n=205 \\
(50.6 \%)\end{array}$ & $\begin{array}{l}\text { 50-64 years old } \\
n=141 \\
(34.8 \%)\end{array}$ & $\begin{array}{l}\geq 65 \text { years old } \\
n=59 \\
(14.6 \%)\end{array}$ & $P$ value \\
\hline Hepatomegaly & $10(4.9)$ & $9(6.4)$ & $2(3.4)$ & 658 \\
\hline Splenomegaly & $68(33.2)$ & $28(19.9)$ & $13(22.0)$ & .015 \\
\hline \multicolumn{5}{|l|}{ Echocardiographic data } \\
\hline Vegetation length & & & & .029 \\
\hline$<10 \mathrm{~mm}$ & $35(17.1)$ & $36(25.5)$ & $18(30.5)$ & \\
\hline$\geq 10 \mathrm{~mm}$ & $146(71.2)$ & $83(58.9)$ & $30(50.8)$ & \\
\hline No vegetation & $24(11.7)$ & $22(15.6)$ & $11(18.6)$ & \\
\hline Vegetation mobility & $84(41.0)$ & $47(33.3)$ & $26(44.1)$ & .238 \\
\hline Moderate or severe valve regurgitation & $29(14.2)$ & $17(12.4)$ & $12(20.3)$ & .547 \\
\hline Moderate or severe valve stenosis & $11(5.4)$ & $27(19.7)$ & $5(8.5)$ & .001 \\
\hline Abscess & $25(12.2)$ & $17(12.1)$ & $8(13.6)$ & .953 \\
\hline Annular abscess & $13(6.4)$ & $10(7.1)$ & $7(11.9)$ & .365 \\
\hline Pseudoaneurysm & $18(8.8)$ & $7(5.0)$ & $2(3.4)$ & .207 \\
\hline Valvular perforation & $32(15.8)$ & $27(19.1)$ & $13(22.0)$ & .480 \\
\hline \multicolumn{5}{|l|}{ Microbiology } \\
\hline Streptococci & $53(25.9)$ & $34(24.1)$ & $13(22.0)$ & .819 \\
\hline Streptococcus viridans & $22(10.7)$ & $9(6.4)$ & $3(5.1)$ & .219 \\
\hline Staphylococci & $40(19.5)$ & $28(19.9)$ & $11(18.6)$ & .981 \\
\hline Staphylococcus aureus & $17(8.3)$ & $9(6.4)$ & $2(3.4)$ & .405 \\
\hline Coagulase-negative staphylococci & $23(11.2)$ & $19(13.5)$ & $9(15.3)$ & .660 \\
\hline Enterococci & $2(1.0)$ & $2(1.4)$ & $1(1.7)$ & \\
\hline Fungi & 0 & $2(1.4)$ & $1(1.7)$ & \\
\hline Polymicrobial & $1(0.5)$ & $1(0.7)$ & 0 & \\
\hline \multicolumn{5}{|l|}{ Complications } \\
\hline Heart failure & $101(49.3)$ & $76(53.9)$ & $37(62.7)$ & .181 \\
\hline Total emboli & $57(27.8)$ & 45 (31.9) & $23(39.0)$ & .247 \\
\hline Emboli under treatment & $60(29.3)$ & $35(24.8)$ & $12(20.3)$ & .339 \\
\hline Intracranial infection & $12(5.9)$ & $4(2.8)$ & $1(1.7)$ & .227 \\
\hline Cerebral emboli & $31(15.1)$ & $29(20.6)$ & $14(23.7)$ & .219 \\
\hline Cerebral hemorrhage, & $14(6.8)$ & $7(5.0)$ & $2(3.4)$ & .544 \\
\hline Arrhythmia & $24(11.7)$ & $33(23.4)$ & $23(39.0)$ & $P<0.001$ \\
\hline Atrial fibrillation & $15(7.3)$ & $29(20.6)$ & $20(33.9)$ & $P<0.001$ \\
\hline Apparition of atrioventricular block & $4(2.0)$ & $4(2.8)$ & $4(6.8)$ & .155 \\
\hline Hepatic insufficiency & $43(21.0)$ & $22(15.6)$ & $6(10.2)$ & .119 \\
\hline Renal insufficiency & $34(16.6)$ & $29(20.6)$ & $18(30.5)$ & .061 \\
\hline \multicolumn{5}{|l|}{ Surgery and mortality } \\
\hline Surgical indication & $204(99.5)$ & $140(99.3)$ & $57(96.6)$ & .142 \\
\hline Surgery indicated and performed & $128(62.4)$ & $83(58.9)$ & $24(40.7)$ & .012 \\
\hline \multicolumn{5}{|l|}{ Reason of no surgery } \\
\hline Medical treatment & $61(80.3)$ & $46(80.7)$ & $23(69.7)$ & \\
\hline Death before surgery & $12(15.8)$ & $11(19.3)$ & $10(30.3)$ & .216 \\
\hline Patient's refusal & $3(3.9)$ & 0 & 0 & \\
\hline In-hospital mortality & $16(7.8)$ & $15(10.6)$ & $12(20.3)$ & $.023^{* *}$ \\
\hline
\end{tabular}


Table 1 Clinical characteristics of patients with infective endocarditis (Continued)

\begin{tabular}{|c|c|c|c|c|}
\hline Variables $\%$ or Mean \pm SD & $\begin{array}{l}<50 \text { years old } \\
n=205 \\
(50.6 \%)\end{array}$ & $\begin{array}{l}50-64 \text { years old } \\
n=141 \\
(34.8 \%)\end{array}$ & $\begin{array}{l}\geq 65 \text { years old } \\
n=59 \\
(14.6 \%)\end{array}$ & $P$ value \\
\hline One-year mortality & $18(8.8)$ & 15 (10.6) & $12(20.3)$ & .044 \\
\hline Cause of death & & & & .124 \\
\hline Heart failure & 0 & $5(33.3)$ & $3(25.0)$ & \\
\hline Sepsis & $10(55.6)$ & $6(40.0)$ & $5(41.7)$ & \\
\hline Cerebral hemorrhage Brain palsy & $6(33.3)$ & $1(6.7)$ & $2(16.7)$ & \\
\hline Life-threatening arrhythmias & $1(5.6)$ & $1(6.7)$ & $1(8.3)$ & \\
\hline Others & $1(5.6)$ & $2(13.3)$ & $1(8.3)$ & \\
\hline
\end{tabular}

$I Q R$ interquartile range, IE Infective endocarditis

${ }^{*} p=0.051:<50$ years group vs $\geq 65$ years group $p=0.526: 50-64$ years group vs $\geq 65$ years group

** $p=0.006<50$ years group vs $\geq 65$ years group $p=0.06750-64$ years group vs $\geq 65$ years group

symptoms before diagnosis, time interval from diagnosis to surgery and time interval from cerebral embolism to diagnosis. Prosthetic valve IE was more common in older patients and native valve IE was more common in younger patients but there were no statistically difference. Old patients with IE had more frequent nosocomial origin $(P=0.007)$.

It was more common for old IE patients to have previous cardiac surgery $(P=0.001)$ and degenerative heart disease $(P=0.001)$ but less common in congenital heart disease $(\mathrm{P}<0.001)$. There was no statistically significant difference between the age groups in terms of fever, anemia, heart murmur, extracardiac IE signs (Osler's nodes, Janeway lesions and nailbed bleeding) and hepatomegaly. However, splenomegaly was less frequent in old patients. Old patients tend to be more frequent with a bad oral hygiene $(p=0.008)$.

The most frequent isolated pathogens in the old groups was streptococci and coagulase-negative staphylococci. Staphylococcus aureus tended to be less frequent with age but it presented no statistically difference. Concerning complications, no difference was observed between groups in heart failure, emboli under treatment, intracranial infection, cerebral emboli, cerebral hemorrhage, hepatic insufficiency and renal insufficiency, except atrial fibrillation, which was more frequent in old subjects.

In-hospital mortality rates were $7.8 \%$ (<50 years group), $10.6 \%$ (50-65 years group) and $15.7 \%$ ( $\geq 65$ years group) ( $p=0.023$ ). In-hospital mortality was high in the $\geq 65$ years group than in the $<50$ years group $(p=0.006)$. In the $\geq 65$ years group, the predominant cause of deaths were sepsis $(41.7 \%)$ and heart failure (25.0\%).

According to the ESC guidelines, surgery was theoretically indicated in 99.5, 99.3 and $96.6 \%$, but was ultimately performed in $62.4,58.9$ and $40.7 \%$ in the $<50$ years, 50-64 years and $\geq 65$ years groups, respectively. The main reason for elder patients not operated was the choice of medical treatment after considering the high comorbidities, high operative risk or multidisciplinary decision. Owing to death before surgery tended to be more frequent with age (15.8 to $19.3 \%$ and $30.3 \%$ ) but there was no statistically difference.

\section{The risk factors for one-year mortality in old patients}

A one-year cox survival analysis was performed for the $\geq 65$ years group. The results were show in Tables 2 and 3 . Significant variables included man, hemodialysis, renal insufficiency, pulmonary arterial hypertension, Pitt score $\geq 4$, vegetation length $>30 \mathrm{~mm}$ and surgical treatment were risk factors for one-year mortality. Surgical treatment $[\mathrm{HR}=$ $2.45,95 \%$ CI $1.027-10.598, P=0.009$ ) was a significant predictor of one-year mortality even after adjusting for confounder. The Kaplan-Meier survival curves revealed that cumulative one-year survival rate was significantly higher in old patients when surgery operated than those not (95.8\% vs $68.6 \%, P=0.007$ ) (Fig. 1 ).

\section{Discussion}

This study represents a large cohort of IE from a single center. It showed that older patients with IE had common clinical symptoms, more nosocomial origin, worse oral hygiene than younger patients. The most frequent isolated pathogens in the old groups was Streptococci. Moreover, they presented more comorbidities, more atrial fibrillation as well as more severe prognosis than younger patients. Surgical therapy was less performed in older patients although the theoretical indications for surgery was clear. Those with surgical therapy had better outcome.

\section{The clinical characteristics of older patients with infective endocarditis}

According to the published researches, the clinical features in older patients were few and untypical, which often led to a delay in the diagnosis of IE $[5,10,11]$. While, Jean et al. [12] found older people had a more severe clinical status than younger patients, which lead to the early diagnosis. However, the clinical presentations in old 
Table 2 Cox univariate analysis of one-year mortality in patients $\geq 65$ year old with infective endocarditis

\begin{tabular}{|c|c|c|c|}
\hline Variables $\%$ or Mean \pm SD & $\begin{array}{l}\text { Survival } \\
n=47\end{array}$ & $\begin{array}{l}\text { Mortality } \\
n=12\end{array}$ & $P$ value \\
\hline \multicolumn{4}{|l|}{ Clinical data } \\
\hline Male & $35(74.5)$ & $6(50.0)$ & .267 \\
\hline Length of hospital stay & $25 \pm 21$ & $16 \pm 10$ & .052 \\
\hline $\begin{array}{l}\text { Symptoms before } \\
\text { echocardiography }\end{array}$ & $26 \pm 37$ & $16 \pm 17$ & .355 \\
\hline $\begin{array}{l}\text { Duration of symptoms } \\
\text { before diagnosis, median (IQR), days }\end{array}$ & $\begin{array}{l}24.0(12.0- \\
42.0)\end{array}$ & $\begin{array}{l}29.0(10.3- \\
54.8)\end{array}$ & .799 \\
\hline $\begin{array}{l}\text { Time interval from emboli to } \\
\text { diagnosis }\end{array}$ & $12 \pm 10$ & $11 \pm 11$ & .868 \\
\hline $\begin{array}{l}\text { Time interval from cerebral } \\
\text { emboli to diagnosis }\end{array}$ & $15 \pm 13$ & $12 \pm 12$ & .724 \\
\hline \multicolumn{4}{|l|}{ IE localization } \\
\hline Native valve & $40(85.1)$ & $9(75.0)$ & .518 \\
\hline Prosthetic valve & $6(12.8)$ & $2(16.7)$ & .862 \\
\hline Community origin & $42(89.4)$ & $5(41.7)$ & $P<0.001$ \\
\hline Nosocomial origin & $2(4.3)$ & $4(33.3)$ & $P<0.001$ \\
\hline \multicolumn{4}{|l|}{ Comorbidities } \\
\hline With predisposing cardiac disease & $29(61.7)$ & $6(50.0)$ & .457 \\
\hline Rheumatic heart disease & $8(17.0)$ & $3(25.0)$ & .752 \\
\hline Congenital heart disease & $9(19.1)$ & $1(8.3)$ & .482 \\
\hline Previous cardiac surgery & $10(21.3)$ & $4(33.3)$ & .475 \\
\hline Degenerative heart disease & $6(12.8)$ & 0 & .466 \\
\hline Chronic pulmonary disease & $1(2.1)$ & $1(8.3)$ & .343 \\
\hline Cancer & $2(4.3)$ & 0 & .690 \\
\hline Hemodialysis & $1(2.1)$ & $3(25.0)$ & .007 \\
\hline Liver cirrhosis & & & .713 \\
\hline Hypertension & 18 (38.3) & $7(58.3)$ & .272 \\
\hline Diabetes & $7(14.9)$ & $3(25.0)$ & .565 \\
\hline Immunodepression & 0 & $4(33.3)$ & $P<0.001$ \\
\hline Bad oral hygiene & $22(46.8)$ & $7(58.3)$ & .713 \\
\hline \multicolumn{4}{|l|}{ Symptoms and signs } \\
\hline Anemia & $31(66.0)$ & $7(58.3)$ & .425 \\
\hline Fever & 44 (93.6) & $9(75.0)$ & .046 \\
\hline Splenomegaly & $11(23.4)$ & $2(16.7)$ & .491 \\
\hline Hepatomegaly & $1(2.1)$ & $1(8.3)$ & .516 \\
\hline New or changing heart murmur & 39 (83.0) & $6(50.0)$ & .021 \\
\hline \multicolumn{4}{|l|}{ Complications } \\
\hline Heart failure & $25(53.2)$ & $12(100.0)$ & .106 \\
\hline Total emboli & $16(34.0)$ & $7(58.3)$ & .143 \\
\hline Emboli under treatment & $8(17.0)$ & $4(33.3)$ & .295 \\
\hline Intracranial infection & $1(2.1)$ & 0 & .713 \\
\hline Cerebral emboli & $8(17.0)$ & $6(50.0)$ & .053 \\
\hline Cerebral hemorrhage & $1(2.1)$ & $1(8.3)$ & .404 \\
\hline Arrhythmia & $16(34.0)$ & $7(58.3)$ & .202 \\
\hline
\end{tabular}

Table 2 Cox univariate analysis of one-year mortality in patients $\geq 65$ year old with infective endocarditis (Continued)

\begin{tabular}{llll}
\hline Variables\% or Mean \pm SD & $\begin{array}{l}\text { Survival } \\
n=47\end{array}$ & $\begin{array}{l}\text { Mortality } \\
n=12\end{array}$ & $P$ value \\
\hline Renal insufficiency & $9(19.1)$ & $9(75.0)$ & .001 \\
Hepatic insufficiency & $3(6.4)$ & $3(25.0)$ & .092 \\
Pulmonary arterial hypertension & $15(31.9)$ & $8(66.7)$ & .037 \\
Moderate or severe valve & $10(21.3)$ & $2(16.7)$ & .570 \\
regurgitation & & & \\
Moderate or severe valve stenosis & $4(8.5)$ & $1(8.3)$ & .912 \\
Annular abscess & $5(10.6)$ & $2(16.7)$ & .564 \\
Pseudoaneurysm & $1(2.1)$ & $1(8.3)$ & .289 \\
Valvular perforation & $10(21.3)$ & $3(25.0)$ & .781 \\
Pitt score $\geq 4$ & $1(2.1)$ & $5(41.7)$ & .001 \\
Vegetation length & & & .062 \\
$\leq 10$ mm & $25(53.2)$ & $4(33.3)$ & \\
$>10$ mm<20 mm & $19(40.4)$ & $5(41.7)$ & \\
$\geq 20$ mm $\leq 30$ mm & $3(6.4)$ & $1(8.3)$ & \\
$>30$ mm & 0 & $2(16.7)$ & .039 \\
Microbiology & & & .050 \\
Streptococci & $12(48.0)$ & $1(16.7)$ & .268 \\
Staphylococci & $8(32.0)$ & $3(50.0)$ & .679 \\
Staphylococcus aureus & 0 & $2(33.3)$ & .039 \\
Enterococci & $1(4.0)$ & 0 & \\
Fungi & 0 & $1(16.7)$ & \\
Surgical treatment & $23(48.9)$ & $1(8.3)$ & .018 \\
\hline
\end{tabular}

patients was not significantly different as compared with the younger patients in our study. And the time to diagnosis was not significantly different compared with the younger patients.

In accordance with previous studies, clinical characteristics varied with aging [12-15]. Older patients were more frail, which often lead to more cardiovascular and general comorbidities and complications than younger patients. In our study, the older patients presented more predisposing factors (like previous cardiac surgery history, degenerative heart disease, hypertension, diabetes and so on) contrary to younger patients who frequently presented congenital heart disease. For IE patients, comorbidities and complications increased with ageing, just like the general population.

Different to the published researches, streptococci was the most frequent isolated pathogens in the old groups in our study. This might be owing to the large number of native valve IE and community-acquired IE in old patients. According to the published researches, streptococci was more prevalent among patients with a native valve and community-acquired IE [16]. What's more, the bad oral hygiene among old patients might be another 
Table 3 Cox multivariate analysis of one-year mortality for patients $\geq 65$ year old with infective endocarditis

\begin{tabular}{llll}
\hline Variables & $P$ value & HR & $95 \% \mathrm{Cl}$ \\
\hline Male & .025 & 3.751 & $1.183-11.891$ \\
Hemodialysis & .007 & 6.146 & $1.633-23.124$ \\
Renal insufficiency & .001 & 8.684 & $2.327-32.407$ \\
Pulmonary arterial hypertension & .037 & 3.627 & $1.083-12.153$ \\
Pitt score $\geq 4$ & $<.001$ & 10.589 & $3.284-34.147$ \\
Vegetation length $>30 \mathrm{~mm}$ & .009 & 10.600 & $1.796-62.569$ \\
Surgical treatment & .009 & 2.45 & $1.027-10.598$ \\
\hline
\end{tabular}

IQR interquartile range, $H R$ hazard ratio, $\mathrm{Cl}$ confidence interval

important reason. The microtrauma caused by these everyday activities (like oral hygiene habits) has been identified to induce oral streptococcal bacteraemia [17]. Therefore, a better control for individual oral hygiene and dental status for old patients was important in reducing oral streptococcal infective endocarditis.

In our study, we found the in-hospital mortality rate and one-year mortality rate in older patients was much higher than the younger patients, which was consistent with previous reports $[3,14,15,18,19]$. As reported previously, older adults were prone to require complex care needs and suffer from multiple comorbidities, which made them vulnerable to health-associated exposure and poor outcomes [14, 20-22]. Besides, the lower operative rate in older patients compared with the younger in our cohort may be another important reason for the higher mortality in older patients $[7,13,14]$.

The in-hospital mortality and one-year mortality were lower in older patients in our study compared with previous studies $[5,14,18]$. Léopold Oliver et al. reported that one-year mortality was higher in the $\geq 80$ year-old group (37.3\%) than in the $<65$-year-old group (13\%) and the 65-80-year-old group (19.7\%), indicating that the mortality rate increased with aging [13]. The few number of very old patients in our study (there were only 5 patients who were over 80 years old) may be an important reason. And a larger cohort for older IE patients was suggest in our region in the future.

\section{Surgical therapy and prognosis for patients $\geq 65$ year old with infective endocarditis}

Previous studies reported that older age, renal failure, prosthetic valve endocarditis, neurological deficit, and cerebral emboli were independent risk factors for oneyear mortality in older patients $[4,15]$. In our study we found the independent risk factors for one-year mortality were man, hemodialysis, renal insufficiency, Pitt score $\geq 4$, vegetation length $>30 \mathrm{~mm}$ and surgical treatment. These

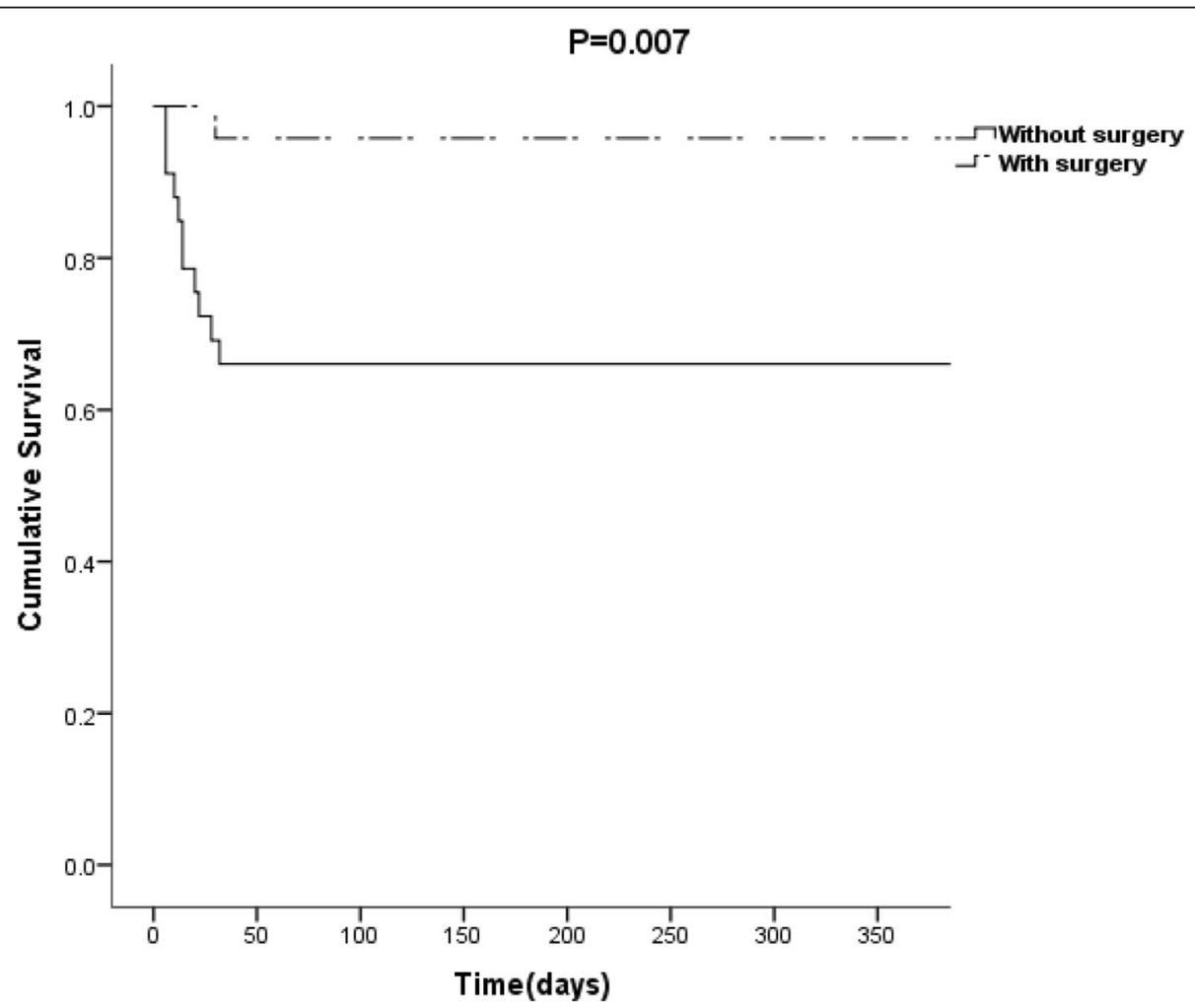

Fig. 1 Figure One-year survival analysis for patients $\geq 65$ years old The Kaplan-Meier survival curves revealed that cumulative one-year survival rate was higher in old patients with surgical intervention than that in patients without surgical intervention $(95.8 \%$ vs $68.6 \%, P=0.007)$ 
events have been confirmed previously to be risk factors for mortality in IE patients [8, 23, 24].

We observed that elder patients with surgical therapy had a lower mortality rate compared with patients not operated during the one-year follow-up. Other recent reports also reached the same conclusion [13]. Surgery was performed less frequent in older patients in our study, although the rate of patients with theoretical indications of surgery was not significantly different compared with the younger. This phenomenon was frequently presented in previous reports $[3,25,26]$. The main consideration may be the increasing risks during the perioperative period owing to the decline in organ function and the presence of comorbidities associated with aging. These factors made the choice of surgical treatment for elderly patients more difficult.

But these considerations could not prevent the old patients with surgical indications from suitable treatments in-time. There are many frailty scores to assess the physical condition of older patients, and some scores showed good reliability in the assessment of mortality independently of age [20]. Some studies have recently proven the utility of these scores for the evaluation of IE-related stroke and prognosis evaluation before cardiac surgery [27]. Therefore, surgery is appropriate in selected old patients with IE. And we suggest a more global patient evaluation and cooperation among multiple specialists to improve IE management in older populations.

\section{Limitations}

There are several limitations in our study. First, it was performed in a referral teaching hospital where most patients were transferred from other medical centers leading to long-term disease and negative blood culture results. Therefore, these results should not be generalized to other patient groups. Second, as a retrospective study, the long-term follow-up was not possible and 29 patients were lost during the one-year follow-up. Finally, the study covered a long period of time in order to keep the enough sample sizes. Changes in treatment regimens and causative organisms could affect the patient prognosis during this period. Therefore, a multiple-center prospective cohort studies conducted in our region was suggested.

\section{Conclusions}

In conclusion, older patients with IE presents more comorbidities, bad oral hygiene, more nosocomial origin and a more severe prognosis than younger patients. Streptococci was the most frequent micro-organisms in this group. Surgery were underused in older patients and those with surgical treatment presented better longterm prognosis.

\section{Abbreviations}

Cl: Confidence interval; HR: Hazard ratio; IE: Infective endocarditis; IQR: Interquartile range; OR: Odds ratios; SD: Standard deviation; TEE: Transesophageal echocardiography; TTE: Transthoracic echocardiography

\section{Acknowledgements}

We are very grateful to the staffs in the Medical record room in the First Affiliated Hospital of Zhejiang University for their support and assistance.

\section{Authors' contributions}

ZZW designed the study and created study protocols, ZZW and YC performed the data collection, data analysis and drafts the manuscript. YHX supervised the study development, helped to review the manuscript and made critical revision to the paper. TTX TSN and QYS performed data collection and helped to review the manuscript. All authors read and approved the final manuscript.

\section{Funding}

This work was partially supported by grants from the Key Research and Development Program of Zhejiang Province (No. 2015C03032). The funder was not involved in the design of the study, data collection, analysis and interpretation of the data and in writing the manuscript.

\section{Availability of data and materials}

The datasets used and analysed during the current study are available from the corresponding author on reasonable request.

\section{Ethics approval and consent to participate}

Ethics approval for this study was submitted and approved through Research Ethics Committee of the First Affiliated Hospital, College of Medicine,

Zhejiang University. The consent to participate is not applicable because the study design is a retrospective data collection. The Research Ethics

Committee of the Hospital was responsible for administrative permissions to access the raw data.

\section{Consent for publication}

Not applicable.

\section{Competing interests}

The authors declare that they have no competing interests.

Received: 22 June 2019 Accepted: 9 October 2019

Published online: 06 November 2019

\section{References}

1. Cahill TJ, Baddour LM, Habib G, Hoen B, Salaun E, Pettersson GB, et al. Challenges in infective endocarditis. J Am Coll Cardiol. 2017;69(3):325-44.

2. K RWSaL. Health care exposure and age in infective endocarditis-results of a contemporary population-based profile of 1536 patients. Eur Heart J. 2010; 31(12):1890-7.

3. Forestier E, Fraisse T, Roubaud-Baudron C, Selton-Suty C, Pagani L. Managing infective endocarditis in the elderly: new issues for an old disease. Clin Interv Aging. 2016;11:1199-206.

4. Gagliardi JP, Nettles RE, McCarty DE, Sanders LL, Corey GR, Sexton DJ. Native valve infective endocarditis in elderly and younger adult patients: comparison of clinical features and outcomes with use of the Duke criteria and the Duke endocarditis database. Clin Infect Dis. 1998;26(5):1165-8.

5. Selton-Suty $C$, Hoen $B$, Grentzinger A, Houplon $P$, Maignan M, Juilliere $Y$, Danchin N, Canton P, Cherrier F. Clinical and bacteriological characteristics of infective endocarditis in the elderly. Heart. 1997;77:260-3.

6. Werner GS, Schulz R, Fuchs JB, Andreas S, Prange H, Ruschewski W, Kreuzer $H$. Infective endocarditis in the elderly in the era of transesophageal echocardiography-clinical features and prognosis compared with younger patients. Am J Med. 1996;100(35):90-7.

7. Durante-Mangoni E, Bradley S, Selton-Suty C, Tripodi MF, Barsic B, Bouza E, Cabell $\mathrm{CH}$, et al. Current features of infective endocarditis in elderly patients. Arch Intern Med. 2008;168(19):2095-103.

8. Linhartova K, Benes J, Gregor P. 2015 ESC guidelines for the management of infective endocarditis. Summary document prepared by the Czech Society of Cardiology. Cor Et Vasa. 2016;58(1):107-28. 
9. Pettersson GB, Coselli JS, Hussain ST, Griffin B, Blackstone EH, Gordon SM, et al. 2016 the American Association for Thoracic Surgery (AATS) consensus guidelines: surgical treatment of infective endocarditis: executive summary. J Thorac Cardiovasc Surg. 2017;153(6):1241-581.

10. Thomas P, Allal J, Bontoux D, Rossi F, Poupet JY, Petitalot JP, et al. Rheumatological manifestations of infective endocarditis. Ann Rheum Dis. 1984;43(5):716-20.

11. Lopez-Wolf D, Vilacosta I, San Roman JA, Fernandez C, Sarria C, Lopez J, et al. Infective endocarditis in octogenarian patients. Rev Esp Cardiol. 2011; 64(4):329-33.

12. Remadi JP, Nadji G, Goissen T, Zomvuama NA, Sorel C, Tribouilloy C Infective endocarditis in elderly patients: clinical characteristics and outcome. Eur J Cardiothorac Surg. 2009;35(1):123-9.

13. Oliver L, Lavoute C, Giorgi R, Salaun E, Hubert S, Casalta JP, et al. Infective endocarditis in octogenarians. Heart. 2017;103(20):1602-9.

14. Lopez J, Revilla A, Vilacosta I, Sevilla T, Villacorta E, Sarria C, et al. Agedependent profile of left-sided infective endocarditis: a 3-center experience. Circulation. 2010;121(7):892-7.

15. Di Salvo G, Thuny F, Rosenberg V, Pergola V, Belliard O, Derumeaux G, et al. Endocarditis in the elderly: clinical, echocardiographic, and prognostic features. Eur Heart J. 2003;24(17):1576-83.

16. Njuguna B, Gardner A, Karwa R, Delahaye F. Infective endocarditis in lowand middle-income countries. Cardiol Clin. 2017;35(1):153-72.

17. Tubiana S, Blotiere PO, Hoen B, Lesclous P, Millot S, Rudant J, et al. Dental procedures, antibiotic prophylaxis, and endocarditis among people with prosthetic heart valves: nationwide population based cohort and a case crossover study. BMJ. 2017;358:3776.

18. Netzer RO, Zollinger E, Seiler C, Cerny A. Native valve infective endocarditis in elderly and younger adult patients: comparison of clinical features and outcomes with use of the Duke criteria. Clin Infect Dis. 1999;28(4):933-5.

19. López-Wolf D, Vilacosta I, San Román JA, Fernández C, Sarriá C, López J, et al. Infective endocarditis in octogenarian patients. Rev Esp Cardiol (English Edition). 2011;64(4):329-33.

20. Sundermann SH, Dademasch A, Seifert B, Rodriguez Cetina Biefer H, Emmert MY, Walther T, et al. Frailty is a predictor of short- and mid-term mortality after elective cardiac surgery independently of age. Interact Cardiovasc Thorac Surg. 2014;18(5):580-5.

21. Fried LP, Tangen CM, Walston J, Newman AB, Hirsch C, Gottdiener J, et al. Frailty in older adults: evidence for a phenotype. J Gerontol A Biol Sci Med Sci. 2001;56(3):146-56.

22. McMillan GJ, Hubbard RE. Frailty in older inpatients: what physicians need to know. Qjm. 2012;105(11):1059-65.

23. Samol A, Kaese S, Bloch J, Gorlich D, Peters G, Waltenberger J, et al. Infective endocarditis on ICU: risk factors, outcome and long-term follow-up. Infection. 2015;43(3):287-95.

24. Nucifora G, Badano LP, Viale P, Gianfagna P, Allocca G, Montanaro D, et al. Infective endocarditis in chronic haemodialysis patients: an increasing clinical challenge. Eur Heart J. 2007;28(19):2307-12.

25. Ramirez-Duque N, Garcia-Cabrera E, Ivanova-Georgieva R, Noureddine M, Lomas JM, Hidalgo-Tenorio C, et al. Surgical treatment for infective endocarditis in elderly patients. J Inf Secur. 2011;63(2):131-8.

26. Taradin GG, Vatutin NT, Prendergast BD, Newton JD, Chaus EA, Smyrnova AS. Infective endocarditis in the elderly: the current view of the problem. Ter Arkh. 2016;88(11):128-37.

27. Murai R, Funakoshi S, Kaji S, Kitai T, Kim K, Koyama T, et al. Outcomes of early surgery for infective endocarditis with moderate cerebral complications. J Thorac Cardiovasc Surg. 2017;153(4):831-40.

\section{Publisher's Note}

Springer Nature remains neutral with regard to jurisdictional claims in published maps and institutional affiliations.

Ready to submit your research? Choose BMC and benefit from:

- fast, convenient online submission

- thorough peer review by experienced researchers in your field

- rapid publication on acceptance

- support for research data, including large and complex data types

- gold Open Access which fosters wider collaboration and increased citations

- maximum visibility for your research: over $100 \mathrm{M}$ website views per year

At BMC, research is always in progress.

Learn more biomedcentral.com/submissions 